\title{
Orientation Analysis of Bulk YBCO from Incomplete Neutron Diffraction Data
}

\author{
D. ChateigneR, ${ }^{a *}$ H.-R. WenK ${ }^{a b}$ AND M. PerneT ${ }^{a}$ \\ ${ }^{a}$ Laboratoire de Cristallographie, CNRS, BP166, 38042 Grenoble Cedex 09, France, and ${ }^{b}$ Department of Geology \\ and Geophysics, University of California, Berkeley, CA 94720,USA. E-mail: chateign@labs.polycnrs-gre.fr
}

(Received 8 January 1996; accepted I July 1996)

\begin{abstract}
Highly incomplete pole figures of a magnetic-fieldaligned melt-textured superconducting sample of the $\mathrm{Y}-\mathrm{Ba}-\mathrm{Cu}-\mathrm{O}$ system were measured by neutron diffraction. The orientation distribution of the main superconducting 123 and a minor 211 phase have been derived with the WIMV algorithm. The 123 phase is strongly textured with a (001) maximum around 22 times that of a random distribution (m.r.d.) and a texture index of 30 . There are two orientation components present that have no apparent relationship with the applied magnetic field. The texture of the 211 phase is weak, with a maximum of 1.7 m.r.d. The textures of the two phases appear to be correlated.
\end{abstract}

\section{Introduction}

Under normal circumstances, texture measurements by neutron diffraction are planned long in advance and sufficient data are collected to cover pole figures as completely as possible. One of the advantages of neutron diffraction is that complete pole figures can be measured without intensity corrections. However, at large facilities there are many events that are beyond the control of the user. Hardware problems can delay an experiment. An actual measurement may reveal that a sample needs a longer counting time or one may simply run out of time because the next user is waiting. This means that data sets may be incomplete in a very arbitrary fashion, and it is often not possible to complete the experiment at a later date. The purpose of this work is to demonstrate that, even in such cases, direct methods of texture analysis make it relatively easy to obtain quantitative orientation data. The sample used is a high-temperature superconductor that crystallized in a magnetic field.

\section{Experimental}

A high magnetic field of $7 \mathrm{~T}$ was used in order to align $\mathrm{YBa}_{2} \mathrm{Cu}_{3} \mathrm{O}_{7-\delta}$ crystallites (123 phase) coupled with a melt-textured growth (MTG) heat treatment (de Rango et al., 1991). A powder composite of 123 and $\mathrm{Y}_{2} \mathrm{BaCuO}_{5}$ (211 phase) particles is heated until 123 melts. At this stage, 211 solid particles are present in a $\mathrm{Ba}-\mathrm{Cu}$-rich

\footnotetext{
* Present address: Department of Geology and Geophysics, University of California, Berkeley, CA 94720, USA.
}

liquid phase. The sample was then cooled at a rate of $1^{\circ} \mathrm{h}^{-1}$ and 123 crystallized from the melt. With this slow cooling rate, the first 123 crystallites present in the liquid are expected to orient in the magnetic field and promote the global texture of the final sample. The resulting sample is an $8 \mathrm{~mm}$-high cylinder, $16 \mathrm{~mm}$ in diameter. The goal was to determine the orientation of 123 and relate it to the direction of the magnetic field.

Neutron diffraction experiments were carried out at the Institut Laue-Langevin (ILL, Grenoble) high-flux reactor using the position-sensitive detector of the D1B beamline. This detector consists of 400 cells covering an $80^{\circ}$ range in $2 \theta$. We used a $2.523 \AA$ monochromatic wavelength provided by a graphite monochromator. The flux at the sample is of the order of $6 \times 10^{6} \mathrm{n} \mathrm{cm}^{-2} \mathrm{~s}^{-1}$. The sample was mounted on a Eulerian cradle with the cylinder axis parallel to the $\varphi$ axis. The goniometer angle $\varphi$ corresponds to the pole-figure azimuth $\beta$ and the angle $\chi$ to the complement of the pole distance $\alpha$. There is an angle transformation that depends on the positioning of the $\chi$ circle $(\omega)$ (Bunge, Wenk \& Pannetier, 1982).

The sample was the last in a series of texture measurements and time was limited. To obtain optimal information, we designed the following data-collection strategy. According to the processing, the sample was

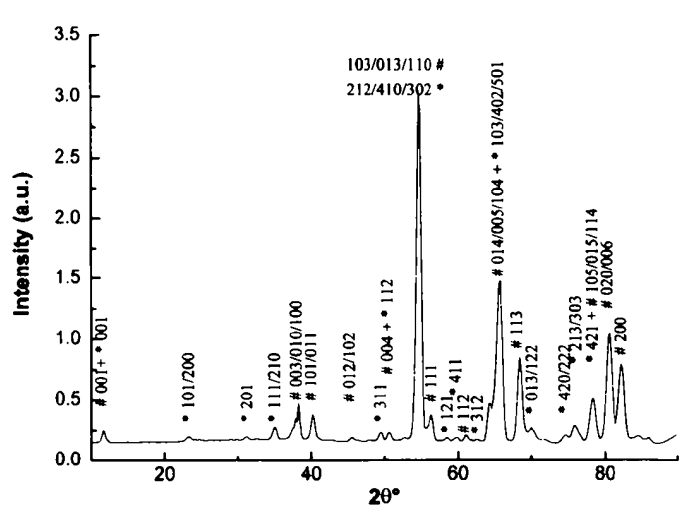

Fig. 1. $2 \theta$ diagram obtained with a position-sensitive detector at the D1B beamline of ILL. This diagram is the summation of the individual 1856 diffraction spectra for all $\backslash \varphi$ positions. Only the $123(\#)$ and $211(*)$ phases are present. 
expected to display a texture with $c$ axes from 123 parallel to the cylinder axis (colinear to the magnetic field axis). It was therefore decided to sample the center of the pole figure at small increments $\left(2.5^{\circ}\right.$ in $\chi$ and $5^{\circ}$ in $\varphi)$ and in a suitable $\omega$ position $\left(10^{\circ}\right)$ to obtain coverage for 001 . When no strong intensities were observed, the strategy was changed and we started to measure from the periphery of the pole figure $\left(0^{\circ}\right)$ inwards with $\omega=$ $25^{\circ}$ corresponding to the medium range of the spectrum. At $\chi=30^{\circ}$, the experiment was aborted at spectrum number 1856. Measuring time for each spectrum was $15 \mathrm{~s}$. We therefore had very incomplete coverage with a small area in the center and a $30^{\circ}$ annulus at the periphery.

When we began to analyse the data, we noticed that the fine coverage was excessive and decided to reduce the very large number of data by averaging two adjacent spectra (in $\varphi$ ), resulting in a total of 928 spectra at different angular positions.

In a first step of data reduction, a diagram summed over all the $1856(\chi, \varphi)$ positions was prepared and used to refine the $\theta$ positions and widths of the peaks of interest and their background level, using the program XRFIT (Rodriguez, Anne \& Pannetier, 1992). The peak profiles were assumed to be Gauss functions. Then, these parameters were fixed in order to cyclically refine integrated intensities at all positions of the sample for each selected diffraction peak. Partial overlaps between peaks were deconvoluted during this stage of data treatment.

For each measuring point and diffraction peak, diffractometer coordinates were converted to polefigure coordinates and entered into boxes on the pole figure. The highly incomplete pole figures were then used to calculate the orientation distribution with the
Berkeley texture package (BEARTEX; Wenk, Matthies \& Donovan, 1996) and the WIMV algorithm (Matthies \& Vinel, 1982).

The 123 phase crystallizes in the orthorhombic Pmmm structure but was treated as tetragonal ( $a=3.85$ and $c=$ $11.66 \AA$ ). Six incomplete pole figures $001,101,102,111$, 112 and 006 were extracted from measurements. The 006 pole figure was assumed to be a superposition of $27 \%$ of 006 and $73 \%$ of 020 . For the minor orthorhombic 211 phase $(a=12.181, b=5.658$ and $c=7.132 \AA$, Pnma), the three incomplete pole figures 201, 121 and 411 were used.

\section{Results}

Fig. 1 represents the summed $2 \theta$ diagram of the 1856 measured spectra. Since the coverage of the pole figure was incomplete and was not done with an equal area scheme, this diagram is not equivalent to that of a random powder. There are strong reflections for the 123 phase and weak ones for 211. An attempt was made to determine the texture of both phases.

At first, we evaluated the orientation-space coverage using the program MIMA in BEARTEX for the two phases, taking into account the extent of the analyzed regions of the pole figures (Table 1). This analysis is based on the concept of the minimum pole density set (MPDS; Vadon, 1981) or minimal pole-figure range (MPR; Helming, 1991), which defines as a necessary condition that the number and range of pole figures must be large enough such that any crystal orientation can be determined unambiguously. The analysis of Helming (1991) indicates that for five pole figures of the 123 phase the minimal range is $71<\alpha<90^{\circ}$ and that

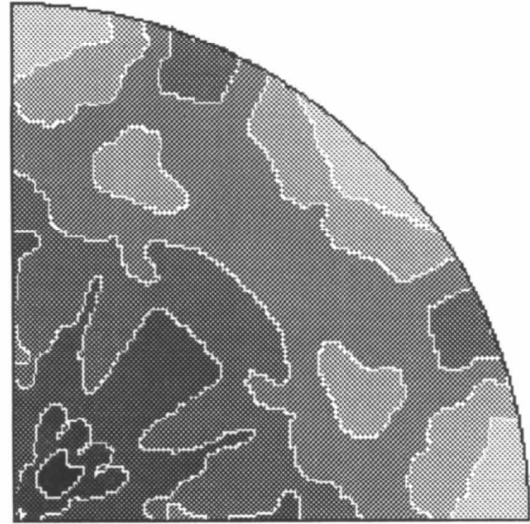

(a)

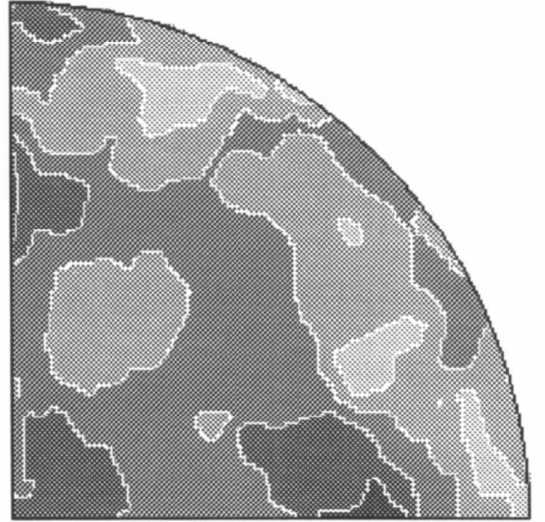

(b)

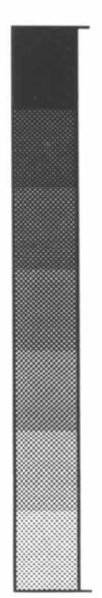

14 max.

\section{$4 \mathrm{~min}$.}

Fig. 2. Plot in crystal reference with the evaluation of the orientation-space coverage for the analyzed pole-figure zones of Table 1 for $(a)$ the 123 and $(b)$ the 211 phases. The levels are the number of orientation paths that pass through each ODF cell. In the case of full azimuthal-ring data coverage, the determinacy can be completely represented on an inverse pole figure. Notice that all the cells in orientation space are determined by at least four paths. (001) is in the left corner; (100) to the right. (Linear scale, equal-area projection.) 
for three pole figures of the 211 phase it is $56<\alpha$ $<90^{\circ}$. Our measurements exceed this condition (Table 1). In the case of discrete methods, a necessary but not sufficient condition is that at least three projection paths from pole figures pass through every orientationdistribution-function (ODF) cell. Since full azimuthal rings have been measured, this condition can be com- pletely analyzed and represented in a crystal coordinate plot. Results of numbers of intersections for the 123 and 211 phases are given in Figs. $2(a)$ and $(b)$, respectively. Values must be at least three in all ODF cells to find a solution for the ODF. This is also satisfied for both phases, with values ranging from 4 to 14 for 123 and 4 to 10 for 211 , although the minima are near the limit

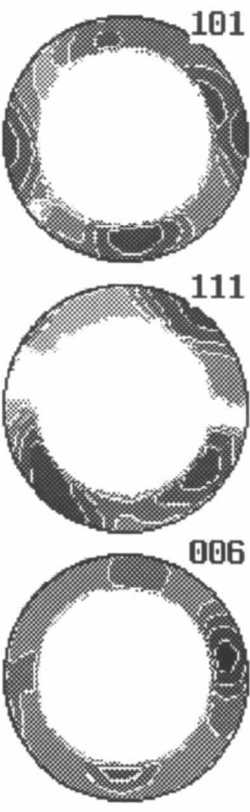

Exp.
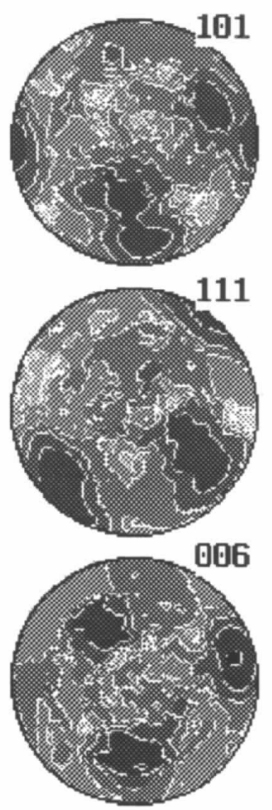

Recalc.
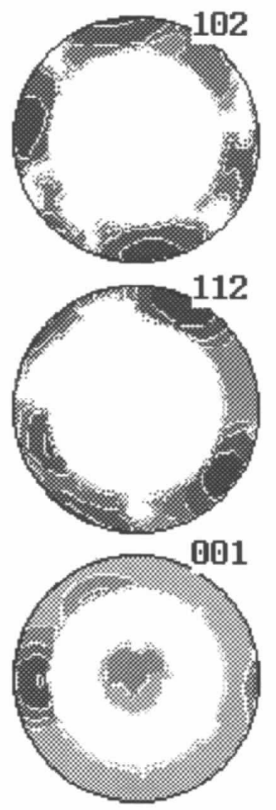

Exp.
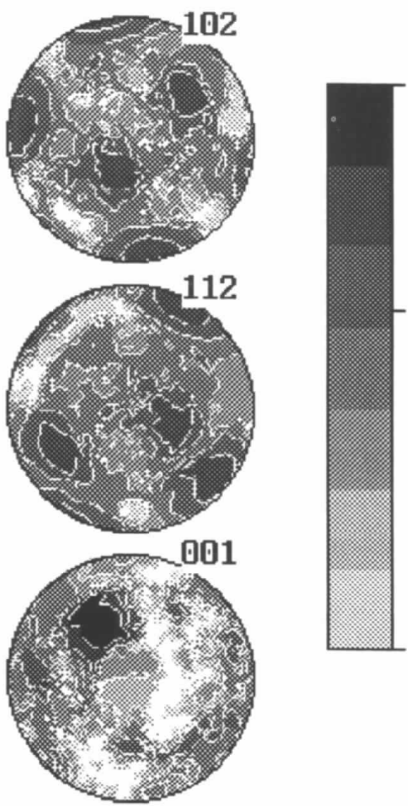

22.35

1 m.r.d.

Fig. 3. Normalized experimental and WIMV recalculated pole figures of the 123 phase. White areas in the experimental pole figures are the nonanalyzed regions. Complete pole figures are recalculated from the ODF. The outer part of the 001 pole figure was not used for model 1. The sample cylinder axis is in the center of pole figures. (Logarithmic intensity scale, equal-area projection.)

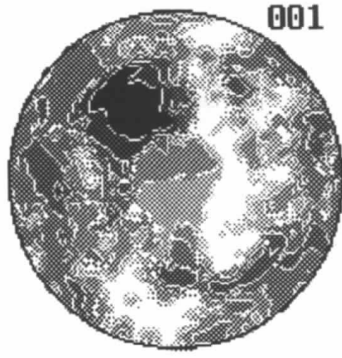

(a)

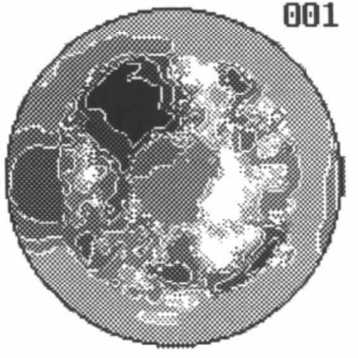

(b)

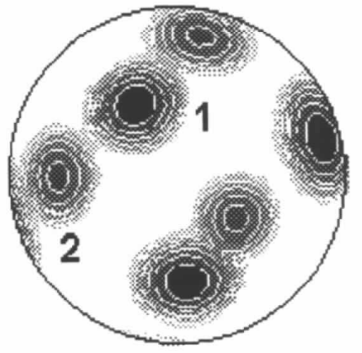

(c)

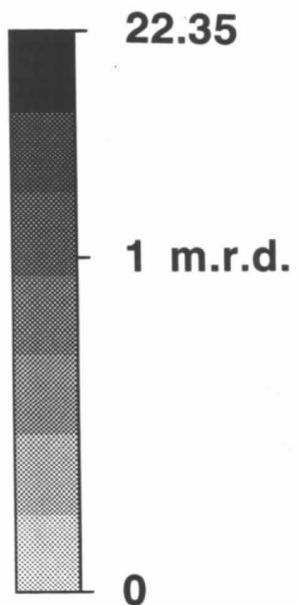

Fig. 4. Recalculated 001 pole figures of the 123 phase when the outer part of the 001 pole figure is $(a)$ not used and $(b)$ used for the ODF calculation. The two texture components 1 (major) and 2 (minor) are illustrated on the combined 001,010 and 100 pole figure $(c)$ recalculated from the ODF components. (Logarithmic intensity scale, equal-area projection.) 
Table 1. Experimental ranges of analyzed pole figures for the two phases 123 and 211

For each $\alpha$ position, the entire $\varphi$ rings have been measured. Notice the highly incomplete analyzed zones.

\begin{tabular}{ccrcc}
\multicolumn{2}{c}{123} & & \multicolumn{2}{c}{211} \\
\cline { 1 - 2 } \cline { 5 - 5 }$h k l$ & $\alpha\left(^{\circ}\right)$ & & $h k l$ & $\alpha\left({ }^{\circ}\right)$ \\
001 & $0-20,65-90$ & & 201 & $0-15,55-90$ \\
101 & $65-90$ & & 121 & $15-25,55-90$ \\
102 & $65-90$ & & 411 & $15-30,55-90$ \\
111 & $65-90$ & & \\
112 & $65-90$ & & \\
$006+020$ & $65-90$ & &
\end{tabular}

and the ODF analysis is therefore not expected to be exceedingly quantitative.

Next, the ODF was calculated, and from it complete pole figures were recalculated. The refinement converges to a unique solution. A comparison of measured and recalculated pole figures for the 123 phase is displayed in Fig. 3. The WIMV program in BEARTEX, which is applicable to all crystal symmetries and triclinic sample symmetry, accepts pole figures consisting of a single ring of data. If two rings are present in a pole figure, they have to be entered as two separate pole figures and the experimental correlation between the two rings is lost. ODFs for two assumptions were calculated: in the first (model 1) we did not use the outer part of the 001 pole figure (results are shown in Fig. 3); in the second we did (model 2). Recalculated 001 pole figures for the two cases are shown, respectively, in Figs. 4(a) and (b). A high orientation density is obtained, with a maximum of 22 m.r.d. on the 001 pole figure, for both calculations. Deviations between the two models are an expression of poor statistics that limit the resolution of the ODF. In general, a fair agreement between observed and recalculated pole figures in the high-density regions is observed, as evidenced by the low RP1 factor (Matthies, 1991) (Table 2). The agreement is less satisfactory for weak-pole-density regions (high RP0 factors). The texture index (Bunge, 1982), which is a measure of the overall texture strength, is high.

Results for the 123 phase were quite surprising. From the measurements, particularly of the 001 pole figure
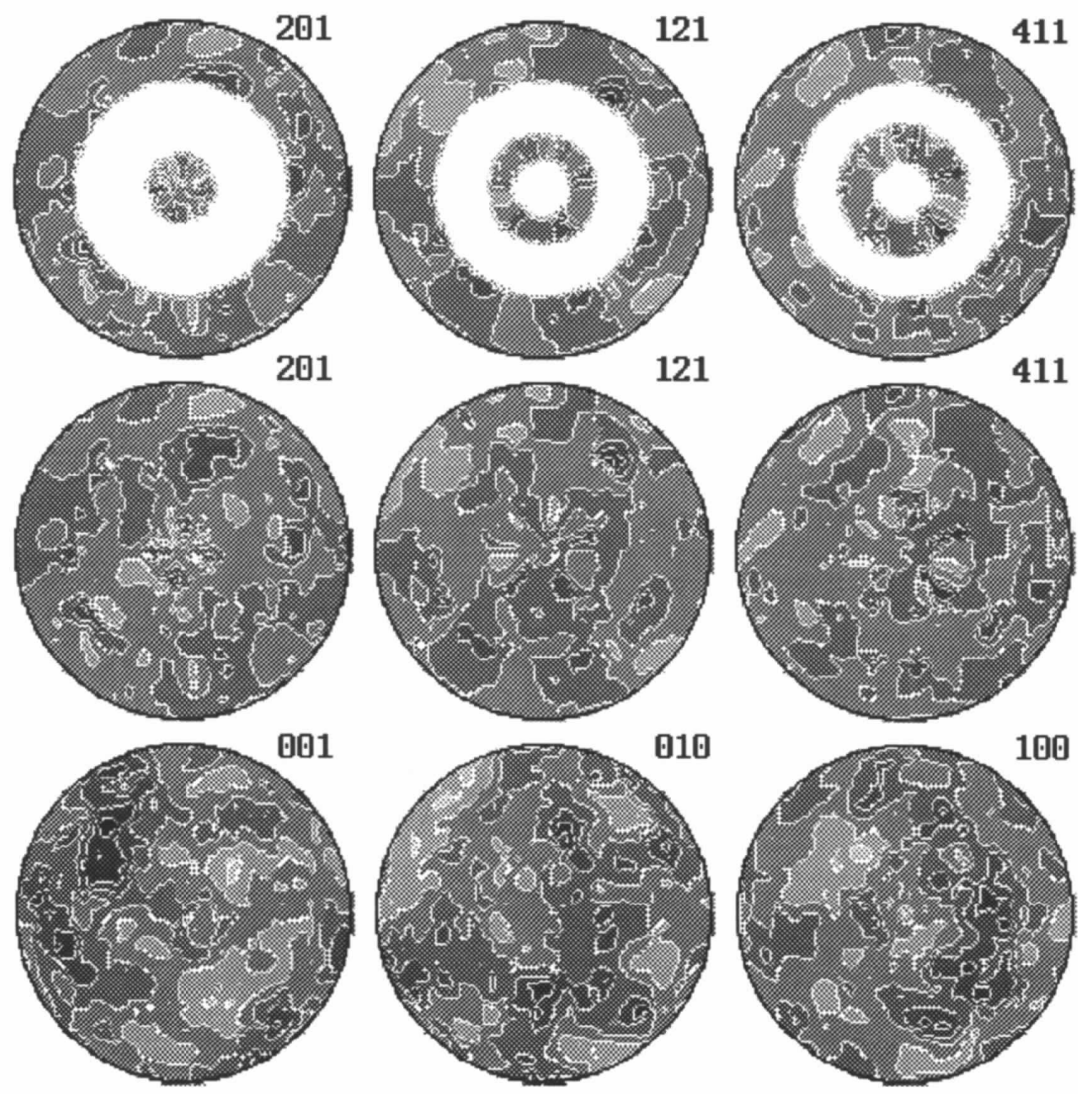

(a)
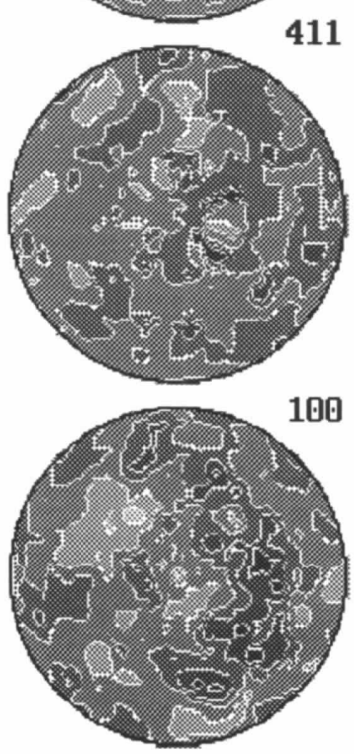

(b)

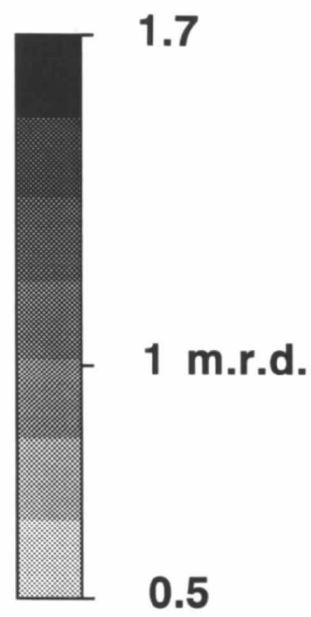

(c)

Fig. 5. (a) Normalized experimental and (b) WIMV recalculated pole figures of the 211 phase. The texture is very weak, with a maximum of only 1.7 m.r.d. (c) Recalculated 001,010 and 100 pole figures of the 211 phase show a preference for [001] directions to be aligned parallel to [001] of component 1 of 123 . (Linear intensity scale, equal-area projection.) 
Table 2. Averaged RP factors (over all the analyzed pole figures) and texture index obtained after WIMV calculation using the analyzed regions of Table $I$

$\begin{array}{lccc} & \text { RP0 }(\%) & \text { RP1 }(\%) & \text { Texturc index } \\ 123(001 \text { center }+ \text { border }) & 27 & 17 & 33 \\ 123(001 \text { center only) } & 24 & 16 & 30 \\ 211 & 2.6 & 2.5 & 1.2\end{array}$

(Fig. 3), the main $c$-axis concentration was expected to be at high $\alpha$ angles, near $70^{\circ}$. It turns out that this is a minor component of the texture (component 2 in Fig. $4 c$ ), with a maximum of only 3.9 m.r.d. for model 1 and 2.3. m.r.d. for model 2. In 001 pole figures, the main $c$-axis component is at $\alpha=45^{\circ}(22.4$ versus 21.9 m.r.d., respectively). Both components are relatively broad and irregular in shape, with full widths at half-maximum of approximately $30^{\circ}$. The components are easily identified in the ODF and are located at $\alpha_{1}$ $=120, \beta_{1}=40$ and $\gamma_{1}=70^{\circ}$ and $\alpha_{2}=180, \beta_{2}$ $=60$ and $\gamma_{2}=70^{\circ}$ (using the Roe/Matthies Euler angles; Matthies, 1990). The components were then expressed as Gaussians with $15^{\circ}$ width and weights corresponding to their observed orientation densities. From this, combined 001,010 and 100 pole figures were calculated (program STPF of BEARTEX), as shown in Fig. $4(c)$, on which the complete orientation is easily recognized. Component 1 is well defined. The strength of the weaker component depends on model assumptions and a quantitative assessment seems to be at the limit of resolution. The components appear to represent a typical growth texture with a divergence of crystallites. From the bulk texture data, it cannot be decided whether one region of the sample is orientation 1 and another one orientation 2 or if the two are intergrown. It was surprising to find that neither of the orientations appeared to have any relation to the applied magnetic field (de Rango et al., 1991).

Fig. 5 presents the results for the texture analysis of the 211 phase. This analysis was performed using the 201, 121 and 411 pole figures (Table 1). Since the 121 and 411 reflections are located in the high $2 \theta$ range, blind areas in the center of the experimental pole figures are extended, which is not the case for the 201 pole figure. From the ODF, the 001,010 and 100 pole figures were recalculated (third line of pole figures of Fig. 5). As denoted by the RP factors, the reliability of the refinement is fair and the recalculated pole figures are in good agreement with the experimental ones.

The orientation analysis was far more difficult, since the three pole figures used are weak, barely above background. About half of the integrated intensities are within one standard deviation. The highest value (maximum in the 121 pole figure) is eight times the standard deviation. The WIMV refinement provides a weak texture with a maximum of 1.7 m.r.d. Interestingly, the 001 maximum for 211 is in a similar position to that for the major 123 component, even though there is no possible crystallographic relationship between the two phases. This observation is in agreement with previous studies (Durand et al., 1995), yet in that case the 211 orientation was attributed to the influence of the magnetic field. However, the shapes of all the 211 particles are not equiaxed, with grains of needlelike shapes, and geometrical effects could influence the growth of 123 platelets.

\section{Conclusions}

A neutron-diffraction texture investigation of one bulk $\mathrm{Y}-\mathrm{Ba}-\mathrm{Cu}-\mathrm{O}$ melt-textured superconductor aligned under high magnetic field has been carried out for both 123 and 211 phases.

The use of direct methods, particularly the WIMV algorithm, was very efficient in the determination of the orientation distribution from a highly incomplete polefigure coverage. It was possible to determine an ODF for the dominant 123 phase and for the minor 211 phase. The possible variation width of feasible ODFs that are consistent with limited data could be further analyzed with indicator functions that have been recently introduced in texture analysis (Schaeben, 1994). The derived orientations show strong alignment of crystallites of the main 123 phase in two arbitrary orientations. These orientations appear to have no relationship to the applied magnetic field. In the case of the minor 211 phase with weak diffraction peaks, a texture has been determined, which is related to the main component of the 123 phase

The experiment illustrates that, with modern methods of quantitative texture analysis, important orientation information can be obtained even if measurements cannot be concluded as planned. In our case, the data collection was limited by access to the neutron beam. In other cases, pole-figure coverage may be limited by principles of the experiment such as X-ray or electron diffraction on very small sample areas of thin films and multilayers and a similar approach to estimate the ODF may be necessary.

The authors appreciate the opportunity to do texture measurements at ILL. They greatly acknowledge $X$. Chaud and E. Beaugnon from Laboratoire EPMMATFORMAG, CNRS Grenoble, who provided the samples, and B. Ouladdiaf from ILL for his help in processing data.

\section{References}

Bunge, H. J. (1982). Texture Analysis in Materials Science. London: Butterworths.

Bunge, H. J., Wenk, H. R. \& Pannetier, J. (1982). Textures Microstruct. 5, 153-170.

Durand, L., Kircher, F., Régnier, P., Chateigner, D., Pellerin, N., Gotor, F. J., Simon, P. \& Odier, P. (1995). Supercond. Sci. Technol. 8, 214-218. 
Helming, K. (1991). Textures Microstruct. 14-18, 187-192.

Matthies, S. (1991). Textures Microstruct. 14-18, 1-12.

Matthies, S. \& Vinel, G. W. (1982). Phys. Status Solidi B, 112, K111.

Rango, P. de, Lees, M., Lejay, P., Sulpice, A., Tournier, R., Ingold, M., Germi P. \& Pernet, M. (1991). Nature (London), 349, $770-772$.
Rodriguez, J., Anne, M. \& Pannetier, J. (1992). STRAP: a System for Time-Resolved Data Analysis (Powder Diffraction Patterns). ILL Internal Report, Grenoble, France.

Schaeben, H. (1994). Mater. Sci. Forum, 157-162, 423-430.

Vadon, A. (1981). Thesis. Université de Metz, France.

Wenk, H. R., Matthies, S. \& Donovan, J. (1996). BEARTEX. Proc. ICOTOM 11. Int. Acad. Publ. Beijing, 212-217. 